
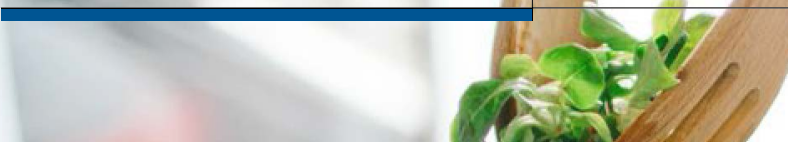

\section{Mit Ernährungsumstellung den Blutdruck senken - das funktioniert!}

\begin{abstract}
Ein zu hoher Blutdruck lässt sich auch ohne Medikamente in den Griff bekommen. Wie das gelingen kann, macht eine auf dem amerikanischen Kardiologen-Kongress präsentierte randomisierte Studie deutlich.
\end{abstract}

ute Nachrichten für Hypertoniker. Eine angemessene Blutdruckkontrolle kann in vielen Fällen durch eine alleinige Ernährungsumstellung gelingen. Einer aktuellen Studie mit 412 Probanden zufolge sind dafür zwei Maßnahmen notwendig: Man muss den Salzanteil in der Ernährung reduzieren und sich nach den Vorgaben der sogenannten DASH-Diät („Dietary Approaches to Stop Hypertension") ernähren.

\section{Blutdrucksenkung ohne Medikamente}

Beides in Kombination hat in der methodisch vorbildlich durchgeführten Studie bei Menschen mit einem leicht bis moderat erhöhtem Blutdruck (120/80 bis $159 / 95 \mathrm{mmHg}$ ) zu einer beträchtlichen Blutdrucksenkung geführt, ohne dass hierfür blutdrucksenkende Medikamente vonnöten waren.

Die Studienergebnisse wurden auf dem Kongress der „American Heart Association" (AHA) präsentiert und zeitgleich im „Journal of the American College of Cardiology“ (JACC) publiziert.

„Die Ergebnisse legen nahe, dass die meisten Erwachsenen mit unkontrolliertem Blutdruck eine bedeutsame Blutdrucksenkung allein durch eine Ernährungsumstellung erreichen können“, resümieren die Studienautoren um Prof. Stephen Juraschek vom Beth Israel Deaconess Medical Center aus Boston.

Die Studie führe vor Augen, wie wichtig Lebensstilmaßnahmen für das Ma- nagement von Hypertonie-Patienten sind.

\section{Randomisierter Vergleich}

Im Gegensatz zu vielen anderen Ernährungsstudien hat die Studie von Juraschek und Kollegen einen wichtigen Qualitätsvorteil, ihr randomisiertes Design. Die Teilnehmer erhielten entweder eine DASH-Diät, die reich an Früchten, Gemüse, Vollkornprodukten, Fisch, Nüssen und Geflügel war und wenig rotes Fleisch, Süßigkeiten und gesüßte Getränke enthielt, oder eine für US-amerikanische Verhältnisse typische Diät.

In beiden Gruppen wurde der Salzgehalt in der Ernährung für drei Wochen entweder auf einem niedrigen, mittleren oder auf einem hohen Niveau gehalten $(1,15 \mathrm{~g}, 2,30 \mathrm{~g}$ oder $3,40 \mathrm{~g}$ Natrium pro Tag) und in der Folge im Sinne eines crossover-Designs nach einer fünftägigen Auswaschphase geändert. Die mittlere Natrium-Dosis entspricht dem aktuell von der AHA empfohlenen Grenzwert.

\section{Effekt der Salzreduktion bei hohen Blutdruck größer}

Die größten Effekte erzielten die Teilnehmer, die zu Studienbeginn den höchsten Blutdruck aufgewiesen hatten.

So fiel der systolische Blutdruck im Falle eines Ausgangsblutdrucks von $\geq 150 \mathrm{mmHg}$ im Schnitt um $20 \mathrm{mmHg}$, wenn die Probanden ihren KochsalzKonsum auf $3 \mathrm{~g}$ (entspricht 1,15g Natri- um) beschränkten und sich im Sinne der DASH-Diät ernährten.

\section{Bei $\geq 150 \mathrm{mmHg}$ war der Effekt besonders groß}

Bei diesen Patienten habe man durch eine Ernährungsumstellung sogar eine größere Blutdrucksenkung erreicht, als man es in Studien mit den üblichen blutdrucksenkenden Medikamenten sehe, berichten Juraschek und Kollegen.

Aber auch bei Blutdruckwerten von $<130,130$ bis 139 und 140 bis $149 \mathrm{mmHg}$ nahm der Druck nach Umstellung auf eine salzreduzierte Ernährung und DASH-Diät um entsprechend 5,3 mmHg, 7,5 $\mathrm{mmHg}$ und 9,7 mmHg ab. Die durch eine Ernährungsumstellung erreichte Blutdrucksenkung falle bei Menschen mit einer Prähypertonie zwar geringer aus als bei Personen, die bereits einen manifesten Bluthochdruck haben, machen die Studienautoren deutlich. Aber trotz allem sei die Wirkung wohl noch hoch genug, um damit eine Senkung des kardiovaskulären Risikos zu erreichen.

Eine Reduktion des Salzkonsums hat auch bei den Patienten in der Kontrollgruppe eine signifikante Blutdrucksenkung gebracht (bei steigendem Ausgangsblutdruck um entsprechend $-3,20$, $-8,56,-8,99$ und $-7,04 \mathrm{mmHg}$ ). Das Einhalten der DASH-Diät ohne Beschränkung des Salzkonsums ging ebenfalls mit einer Blutdrucksenkung einher, dieser Effekt war allerdings nicht signifikant $(-4,5,-4,3,-4,7$ und $-10,6 \mathrm{mmHg}$; $\mathrm{p}=0,66)$.

Veronika Schlimpert

Quelle: Kongress der American Heart Association (AHA) 2017, 11.-15.11.2017, Anaheim Juraschek $S$, Miller $E$, Weaver $C$ et al. Effects of Sodium Reduction and the DASH Diet in Relation to Baseline Blood Pressure. J Am Col Cardiol 2017; https://doi.org/10.1016/j.jacc.2017.10.011 